\title{
Investor Attention, Analyst Optimism, and Stock Price Crash Risk
}

\author{
Shuke Shi* \\ School of Finance and Economics, Jiangsu University, Zhenjiang 212013, China \\ *Corresponding author: Shuke Shi, ssk0420@163.com
}

\begin{abstract}
This paper used the A-shares listed companies in China as samples, constructed a comprehensive indicator of investor attention, and conducted an empirical analysis on the correlations among investor attention, analyst optimism, and stock price crash risk. The results indicated that investor attention aggravates the stock price crash risk and has a positive effect on analyst optimism. Meanwhile, the analyst optimism plays a mediating role in the positive correlation between investor attention and stock price crash risk. In addition to that, institutional investor attention also has direct and indirect effects on the crash risk.
\end{abstract}

Keywords: Stock price crash risk; Analyst optimism; Investor attention

Publication date: June 2021; Online publication: June 30, 2021

\section{Introduction}

With the development of China's financial market, the stock price crash risk has increasingly become the focus in the academic circle. Jin and Myers suggested that the behavior of a company's management in hiding negative information would lead to a continuous buildup of the company's bad news and when it reaches a certain critical value, the accumulated hidden information would be released to the market in an intense manner which is likely to aggravate the stock price crash risk. ${ }^{[1]}$ Investors and analysts are important players in the capital market. The emotions of investors have a direct impact on stock prices, which would eventually affect the stability of the financial market. In comparison to ordinary investors, analysts have more advantages in terms of professional knowledge and their access to information. However, due to conflicting interests, analysts generally tend to be optimistic. ${ }^{[2]}$ Their optimism bias can easily mislead investors' decisions, thereby affecting companies' stock prices. Hence, it is necessary to study the relationships among investors, analysts, and the stock price crash risk.

Using samples from China's A-shares listed companies from 2013 to 2019, this paper constructed a comprehensive indicator of investor attention, conducted a regression analysis on the correlations among investor attention, analyst optimism, and stock price crash risk, as well as discussed the mediating role of analyst optimism. This paper further explored the direct and indirect effects of institutional investor attention on stock price crash risk which would serve as a fresh input for the research on investor attention.

This study may have the following contributions. Firstly, differing from other studies that measure investor attention with only a single variable, this paper focused on both the micro and macro perspectives in constructing a comprehensive indicator of investor attention. This would help to enrich the dimension to measure the variable and would reflect investor attention in a more comprehensive manner. Secondly, this article innovatively tested the correlations among three variables and introduced the intermediary effect mechanism to explore the intermediary role of analyst optimism. This would broaden the research concepts in this field. Thirdly, due to the differences between institutional and ordinary investors, the shareholding 
ratio of institutional investors was used as an independent variable to further analyze the correlations among institutional investor attention, analyst optimism, and stock price crash risk which aimed to enrich the research on factors that affect the crash risk.

\section{Theoretical Discussions}

According to the "limited attention" theory, attention is a scarce resource. Investors are more likely to be attracted by extreme events and they would conduct a series of trading behaviors driven by attention. ${ }^{[3][4]}$ Under the influence of irrational emotions, individual investors are more inclined to trade stocks that have higher attention which would then trigger the "herd effect" and result in the rise of stock prices. An excessive rise in prices may easily cause a deviation between stock prices and the real value of a company, thus increasing the potential risk of stock price crash.

Meanwhile, Abreu and Brunnermeier suggested that high investor attention may contribute to the formation of bubbles in the stock market. ${ }^{[5]}$ The speculative mentality of investors would lead to an abnormal rise in stock prices. Hence, this would increase the risk of stock price crash caused by the accumulation of negative information. Based on analyses above, the proposed hypothesis is as follows:

Hypothesis 1: Investor attention can aggravate corporate stock price crash risk.

According to relevant research, in view of diverse brokerages, analysts not only face the pressure of internal research, brokerage, underwriting, and proprietary business, but they also need to maintain relationships with institutional investors and company managements. ${ }^{[6][7]}$ Due to conflicting interests, analysts are more likely to issue earnings forecasts with optimism bias. In a study, Loh concluded that investor attention is positively correlated with immediate reactions in the stock market. ${ }^{[8]}$ Overall, the higher the investor attention, the stronger the impact of analyst optimism on stock prices, thereby helping to meet the demands of stakeholders and facilitating the realization of analyst potential benefits. In regard to that, the proposed hypothesis is as follows:

Hypothesis 2: Investor attention has a significant positive effect on analyst optimism.

Based on the above hypothesis, the higher the investor attention, the stronger the analyst optimism. On the one hand, although positive forecasts may contribute to the rise of stock prices, excessive optimism bias would result in the company's negative news to be hidden, thereby enhancing the company's information opacity. Stock prices then would not reflect the company's true value and the likelihood of a sharp drop in stock prices would increase. On the other hand, forecasts with optimism bias would lead investors to have higher expectations for certain companies. This would then trigger investors to overreact when receiving negative information and intensify earnings management behaviors by the company, thereby aggravating the stock price crash risk. In regard to that, the following hypothesis is proposed:

Hypothesis 3: Analyst optimism plays a mediating role in the positive correlation between investor attention and crash risk.

\section{Sample and Measurement of Variables}

\subsection{Data and sample}

This research used the A-shares listed companies in Shanghai's and Shenzhen's stock markets from 2013 to 2019 as samples and excluded financial firms, firms under special treatment (ST) and *ST status, firms with missing data, and annual trading weeks less than 30. There were 4062 firm-year observations in the finalized sample. All the data used were from China Stock Market \& Accounting Research (CSMAR) and RESSET databases. 


\subsection{Measurement of variables}

\subsubsection{Dependent variable}

Following Kim, Fu, and Wen, this paper used the negative coefficient of skewness (NCSKEW) and downto-up volatility $(D U V O L)$ to measure stock price crash risk. ${ }^{[9][10][11]}$

Firstly, the weekly return data of stock was regressed:

$$
R_{i, t}=\alpha_{i}+\beta_{1} R_{m, t-2}+\beta_{2} R_{m, t-1}+\beta_{3} R_{m, t}+\beta_{4} R_{m, t+1}+\beta_{5} R_{m, t+2}+\varepsilon_{i, t}
$$

$R_{i, t}$ represented the return of stock $i$ in week $t$, and $R_{m, t}$ represented the value-weighted return of China's A-shares market in week $t$. The specific return on stock $i$ in week $t$ was $W_{i, t}=\operatorname{Ln}\left(1+\varepsilon_{i, t}\right)$.

Secondly, the following variables were constructed based on $W_{i, t}$.

(1) Negative coefficient of skewness of returns

$$
N C S K E W_{i, t}=-\left\lfloor n(n-1)^{3 / 2} \sum W_{i, t}^{3}\right\rfloor /\left\lfloor(n-1)(n-2)\left(\sum W_{i, t}{ }^{2}\right)^{3 / 2}\right\rfloor
$$

$n$ indicated the number of trading weeks for stock $i$ in each year.

(2) Down-to-up volatility

$$
D_{U V O L_{i, t}}=\log \left\{\left[\left(n_{u}-1\right) \sum_{D O W N} W_{i, t}^{2}\right] /\left[\left(n_{d}-1\right) \sum_{U P} W_{i, t}{ }^{2}\right]\right\}
$$

$n_{u}$ and $n_{d}$ represented the number of weeks in which stock prices rose and fell, respectively. When the weekly return of stock $i$ was higher than average weekly return of all stocks, the week was defined as a rising week; otherwise, it was defined as a falling week.

\subsubsection{Independent variable}

This study selected various indicators that reflected investor attention based on macro and micro perspectives then, constructed a comprehensive indicator to measure investor attention. At the macro level, this research measured investor attention from two aspects. The first was stock earnings, comprising of $A A R$ and VOLATILITY which directly reflected investors' attention. The second was stock transaction status, comprising of VOLUME and TUR which indirectly reflected investors' attention. At the micro level, this paper focused on the attention of authoritative institutions including IO, ANALYST, and REPORTS that may affect investors' behaviors. Table 1 shows the definition of the indicators. Referring to the methods used by Fama and French, ${ }^{[12]}$ this paper standardized the 7 indicators. When the index of an individual stock was higher than the median of all stocks, it was recorded as 1 , otherwise recorded as 0 . After standardizing the 7 indicators, the average value was taken to define the final indicator ATT. The formula is as following:

$$
A T T_{i, t}=\left(A A R_{i, t}+\text { VOLATILITY }_{i, t}+\text { VOLUME }_{i, t}+T U R_{i, t}+I O_{i, t}+A N A L Y S T_{i, t}+\text { REPORTS }_{i, t}\right) / 7
$$

Table 1. Indicators measuring investor attention

\begin{tabular}{c|c|c|c}
\hline Dimension & Indicator & Symbol & Indicator measurement \\
\hline \multirow{4}{*}{$\begin{array}{c}\text { The macro } \\
\text { perspective }\end{array}$} & Abnormal annual return rate & AAR & The absolute value of annual stock return minus the average \\
& market annual return
\end{tabular}




\subsubsection{Mediating variable}

According to Jackson's method, ${ }^{[13]}$ the analyst optimism was defined using the following formula:

$$
O p t_{i, j, t}=\left(F_{i, j, t}-A_{i, t}\right) / P_{i}
$$

$F_{i, j, t}$ represented the predicted earnings per share (EPS) of company $i$ by analyst $j$ in year $t, A_{i, t}$ represented the actual EPS of company $i$ in year $t$, and $P_{i}$ was the closing stock price of company $i$ on the trading day before the analyst releases earnings forecast. In this paper, the proportion of analysts with $O p t_{i, j, t}$ greater than 0 was recorded as OPTIMISM.

\subsection{Model specification}

This paper used the mediating effect research method to construct the following three regression models:

$$
\begin{aligned}
& \text { CRASHRISK }_{i, t}=\alpha_{0}+\alpha_{1} \times \text { ATT }_{i, t-1}+\delta \times \text { ControlVariables }_{i, t-1}+\varepsilon_{i, t} \\
& \text { OPTIMISM }_{i, t}=\beta_{0}+\beta_{1} \times \text { ATT }_{i, t-1}+\delta \times \text { ControlVariables }_{i, t-1}+\varepsilon_{i, t} \\
& \text { CRASHRISK }_{i, t}=\gamma_{0}+\gamma_{1} \times \text { ATT }_{i, t-1}+\gamma_{2} \times \text { OPTIMISM }_{i, t}+\delta \times \text { ControlVariables }_{i, t-1}+\varepsilon_{i, t}
\end{aligned}
$$

Model (6) was used to test Hypothesis 1, model (7) was constructed to test Hypothesis 2, and model (8) was used to examine Hypothesis 3. If $\alpha 1, \beta 1, \gamma 1$, and $\gamma 2$ were all significant, it indicates that analyst

\begin{tabular}{|c|c|c|}
\hline Variable type & Variable symbol & Variable definition \\
\hline \multirow{2}{*}{ Independent variable } & $A T T$ & A comprehensive indicator of investor attention \\
\hline & $I O$ & Shareholding ratio of institutional investors \\
\hline \multirow{2}{*}{ Dependent variable } & $N C S K E W$ & Negative skewness of weekly stock specific return \\
\hline & $D U V O L$ & Volatility ratio of weekly stock specific return \\
\hline Mediating variable & OPTIMISM & The proportion of analysts with $O p t_{i, j, t}$ greater than 0 \\
\hline \multirow{13}{*}{ Control variable } & TURNOVER & Monthly average excess turnover rate \\
\hline & SIGMA & The standard deviation of weekly firm-specific returns \\
\hline & $R E T$ & The mean of weekly firm-specific returns \\
\hline & $S I Z E$ & Firm's total assets \\
\hline & $B M$ & Book-to-market value ratio \\
\hline & $L E V$ & Asset-liability ratio \\
\hline & $R O A$ & Return on total assets \\
\hline & $A B A C C$ & The absolute value of discretionary accruals \\
\hline & LIQUIDITY & Liquidity ratio \\
\hline & GROWTH & Growth rate of basic earnings per share \\
\hline & STATE & Property dummy variable \\
\hline & $Y E A R$ & Time dummy variable \\
\hline & INDUSTRY & Industry dummy variable \\
\hline
\end{tabular}
optimism has a partial mediating effect between investor attention and stock price crash risk. Descriptions of the research variables are reported in Table 2.

Table 2. Research variables 


\section{Empirical Results}

\subsection{Descriptive statistics}

According to Table 3, there was a significant difference in terms of negative skewness and the volatility ratio of weekly specific returns of the samples indicating that crash risks of different companies were significantly different. The overall analyst optimism and investor attention levels were high.

Table 3. Descriptive statistics

\begin{tabular}{cccccc}
\hline Variable & $\mathrm{N}$ & Mean & $\mathrm{Sd}$ & Min & Max \\
\hline NCSKEW & 4739 & -0.267 & 0.708 & -5.188 & 4.282 \\
DUVOL & 4739 & -0.180 & 0.475 & -2.417 & 2.499 \\
OPTIMISM & 4739 & 0.764 & 0.266 & 0.000 & 1.000 \\
ATT & 4739 & 0.503 & 0.243 & 0.000 & 1.000 \\
TURNOVER & 4739 & -0.016 & 0.291 & -2.697 & 1.513 \\
SIGMA & 4739 & 0.045 & 0.019 & 0.009 & 0.158 \\
RET & 4739 & -0.001 & 0.001 & -0.013 & 0.000 \\
SIZE & 4739 & $3.636 \mathrm{e}+10$ & $1.391 \mathrm{e}+11$ & $4.152 \mathrm{e}+08$ & $2.441 \mathrm{e}+12$ \\
BM & 4739 & 0.638 & 0.266 & 0.071 & 1.407 \\
LEV & 4739 & 0.441 & 0.192 & 0.020 & 0.948 \\
ROA & 4739 & 0.053 & 0.052 & -0.507 & 0.590 \\
ABACC & 4739 & 0.053 & 0.057 & 0.000 & 1.135 \\
LIQUIDITY & 4739 & 2.127 & 2.234 & 0.130 & 48.169 \\
GROWTH & 4739 & 0.079 & 13.123 & -504.659 & 305.349 \\
IO & 4739 & 0.411 & 0.263 & 0.000 & 0.987 \\
\hline
\end{tabular}

\subsection{Regression analysis}

Table 4 shows the regression results of the three models. Test 1 examined the correlation between investor attention and stock price crash risk, Test 2 examined the correlation between investor attention and analyst optimism, and Test 3 tested the mediating effect of analyst optimism.

The results of Test 1 showed that investor attention was significantly positively correlated with crash risk at 5\% and $10 \%$ levels respectively, indicating that investor attention exacerbated the stock price crash risk. Under the influence of irrational emotions, high investor attention would lead to the herd behavior in the market, trigger their overreaction to negative information, and fuel the stock market bubble which tends to cause a rise in stock prices and eventually, aggravate crash risk.

The results of Test 2 showed that regression coefficients between investor attention and analyst optimism were all positive and significant at $1 \%$ level, indicating that there was a significant positive correlation between investor attention and analyst optimism. Due to conflicting interests, high investor attention may fortify the influence that analyst optimism has on immediate market reaction, which increases the company's stock returns and facilitates the realization of analyst potential benefits. Investor attention turned out to be an enhancer for analyst optimism.

The results of Test 3 showed that investor attention still had a significant positive impact on crash risk at $5 \%$ and $10 \%$ levels respectively even after the mediating variable was included. At the same time, analyst optimism was significantly positively correlated with crash risk at $10 \%$ level, indicating that analyst optimism played a partial mediating role in the positive correlation between investor attention and crash risk. Analyst optimism provides an opportunity to easily hide the company's negative information which would result in the deviation between stock prices and the corporate's actual value. Strong optimism bias 
tends to raise investors' expectations, increase the pressure on management, and trigger earnings management behaviors which would lead to potential crash risk.

Table 4. Investor attention, analyst optimism, and crash risk

\begin{tabular}{|c|c|c|c|c|c|c|}
\hline VARIABLES & $\begin{array}{c}(1) \\
\text { Test } 1 \\
\text { NCSKEW } \\
\end{array}$ & $\begin{array}{c}(2) \\
\text { Test } 2 \\
\text { OPTIMISM }\end{array}$ & $\begin{array}{c}(3) \\
\text { Test } 3 \\
N C S K E W \\
\end{array}$ & $\begin{array}{c}(4) \\
\text { Test } 1 \\
D U V O L \\
\end{array}$ & $\begin{array}{c}(5) \\
\text { Test } 2 \\
\text { OPTIMISM } \\
\end{array}$ & $\begin{array}{c}(6) \\
\text { Test } 3 \\
\text { DUVOL }\end{array}$ \\
\hline$A T T_{t-1}$ & $\begin{array}{c}0.058 * * \\
(2.53)\end{array}$ & $\begin{array}{c}0.087 * * * \\
(3.86)\end{array}$ & $\begin{array}{c}0.058 * * \\
(2.50)\end{array}$ & $\begin{array}{c}0.044 * \\
(1.88)\end{array}$ & $\begin{array}{c}0.087 * * * \\
(3.86)\end{array}$ & $\begin{array}{c}0.043 * \\
(1.85)\end{array}$ \\
\hline OPTIMISM & & & $\begin{array}{c}0.033^{*} \\
(1.90)\end{array}$ & & & $\begin{array}{c}0.030^{*} \\
(1.70)\end{array}$ \\
\hline OPTIMISM $_{t-1}$ & & $\begin{array}{c}0.156 * * * \\
(8.88)\end{array}$ & & & $\begin{array}{c}0.156^{* * * *} \\
(8.88)\end{array}$ & \\
\hline$S I G M A_{t-1}$ & & $\begin{array}{c}-0.205 * * * \\
(-2.64)\end{array}$ & & & $\begin{array}{c}-0.205^{* * *} \\
(-2.64)\end{array}$ & \\
\hline$R E T_{t-1}$ & $\begin{array}{c}-0.068 * * * \\
(-2.66)\end{array}$ & $\begin{array}{l}-0.064 \\
(-0.91)\end{array}$ & $\begin{array}{c}-0.071 * * * \\
(-2.80)\end{array}$ & $\begin{array}{c}-0.045^{*} \\
(-1.75)\end{array}$ & $\begin{array}{l}-0.064 \\
(-0.91)\end{array}$ & $\begin{array}{c}-0.048^{*} \\
(-1.88)\end{array}$ \\
\hline$S I Z E_{t-1}$ & & $\begin{array}{c}0.218 * * * \\
(2.60)\end{array}$ & & & $\begin{array}{c}0.218 * * * \\
(2.60)\end{array}$ & \\
\hline$L E V_{t-1}$ & $\begin{array}{l}-0.020 \\
(-0.42)\end{array}$ & $\begin{array}{c}-0.175 * * * \\
(-3.45)\end{array}$ & $\begin{array}{l}-0.015 \\
(-0.31)\end{array}$ & $\begin{array}{l}-0.042 \\
(-0.91)\end{array}$ & $\begin{array}{c}-0.175 * * * \\
(-3.45)\end{array}$ & $\begin{array}{l}-0.038 \\
(-0.81)\end{array}$ \\
\hline $\operatorname{LIQUIDITY}_{t-1}$ & & $\begin{array}{l}0.049 \\
(1.46)\end{array}$ & & & $\begin{array}{l}0.049 \\
(1.46)\end{array}$ & \\
\hline$G R O W T H_{t-1}$ & & $\begin{array}{l}-0.019 \\
(-1.04)\end{array}$ & & & $\begin{array}{l}-0.019 \\
(-1.04)\end{array}$ & \\
\hline$A B A C C_{t-1}$ & $\begin{array}{l}-0.003 \\
(-0.17)\end{array}$ & $\begin{array}{c}0.046^{* * * *} \\
(2.64)\end{array}$ & $\begin{array}{l}-0.004 \\
(-0.21)\end{array}$ & $\begin{array}{l}0.005 \\
(0.25)\end{array}$ & $\begin{array}{c}0.046^{* * * *} \\
(2.64)\end{array}$ & $\begin{array}{l}0.004 \\
(0.22)\end{array}$ \\
\hline$N C S K E W_{t-1}$ & $\begin{array}{c}-0.126 * * * \\
(-7.25)\end{array}$ & & $\begin{array}{c}-0.126 * * * \\
(-7.29)\end{array}$ & & & \\
\hline$T U R N O V E R_{t-1}$ & $\begin{array}{l}-0.027 \\
(-1.37)\end{array}$ & & $\begin{array}{l}-0.028 \\
(-1.38)\end{array}$ & $\begin{array}{l}-0.024 \\
(-1.18)\end{array}$ & & $\begin{array}{l}-0.024 \\
(-1.18)\end{array}$ \\
\hline$B M_{t-1}$ & $\begin{array}{c}-0.292 * * * \\
(-6.06)\end{array}$ & & $\begin{array}{c}-0.293 * * * \\
(-6.10)\end{array}$ & $\begin{array}{c}-0.310 * * * \\
(-6.40)\end{array}$ & & $\begin{array}{c}-0.311 * * * \\
(-6.43)\end{array}$ \\
\hline$R O A_{t-1}$ & $\begin{array}{l}-0.014 \\
(-0.46)\end{array}$ & & $\begin{array}{l}-0.018 \\
(-0.59)\end{array}$ & $\begin{array}{l}-0.026 \\
(-0.84)\end{array}$ & & $\begin{array}{l}-0.029 \\
(-0.95)\end{array}$ \\
\hline$D U V O L_{t-1}$ & & & & $\begin{array}{c}-0.148 * * * \\
(-8.64)\end{array}$ & & $\begin{array}{c}-0.149 * * * \\
(-8.67)\end{array}$ \\
\hline CONSTANT & $\begin{array}{l}-0.123 \\
(-0.95)\end{array}$ & $\begin{array}{l}0.147 \\
(1.17)\end{array}$ & $\begin{array}{l}-0.128 \\
(-0.99)\end{array}$ & $\begin{array}{l}-0.041 \\
(-0.31)\end{array}$ & $\begin{array}{l}0.147 \\
(1.17)\end{array}$ & $\begin{array}{l}-0.045 \\
(-0.35)\end{array}$ \\
\hline $\begin{array}{c}\text { YEAR } \\
\text { STATE } \\
\text { INDUSTRY }\end{array}$ & $\begin{array}{l}\text { Control } \\
\text { Control } \\
\text { Control }\end{array}$ & $\begin{array}{l}\text { Control } \\
\text { Control } \\
\text { Control }\end{array}$ & $\begin{array}{l}\text { Control } \\
\text { Control } \\
\text { Control }\end{array}$ & $\begin{array}{l}\text { Control } \\
\text { Control } \\
\text { Control }\end{array}$ & $\begin{array}{l}\text { Control } \\
\text { Control } \\
\text { Control }\end{array}$ & $\begin{array}{l}\text { Control } \\
\text { Control } \\
\text { Control }\end{array}$ \\
\hline $\begin{array}{c}\text { Observations } \\
\text { R-squared }\end{array}$ & $\begin{array}{l}4,062 \\
0.089\end{array}$ & $\begin{array}{l}4,062 \\
0.066\end{array}$ & $\begin{array}{l}4,062 \\
0.090\end{array}$ & $\begin{array}{l}4,062 \\
0.091\end{array}$ & $\begin{array}{l}4,062 \\
0.066\end{array}$ & $\begin{array}{l}4,062 \\
0.092\end{array}$ \\
\hline
\end{tabular}

$* * * \mathrm{p}<0.01, * * \mathrm{p}<0.05, * \mathrm{p}<0.1$ 


\subsection{Robustness test}

In order to ensure the robustness of these regression results, five indicators (AAR, VOLATILITY, IO, ANALYST and REPORTS) were selected to replace the variable of investor attention. The robustness results of substituting independent variables are reported in Table 5.

Table 5. Robustness results of substituting independent variables

\begin{tabular}{|c|c|c|c|c|c|c|}
\hline VARIABLES & $\begin{array}{c}(1) \\
\text { Test1 } \\
\text { NCSKEW }\end{array}$ & $\begin{array}{c}(2) \\
\text { Test2 } \\
\text { OPTIMISM }\end{array}$ & $\begin{array}{c}(3) \\
\text { Test3 } \\
\text { NCSKEW }\end{array}$ & $\begin{array}{c}(4) \\
\text { Test1 } \\
\text { DUVOL }\end{array}$ & $\begin{array}{c}(5) \\
\text { Test2 } \\
\text { OPTIMISM }\end{array}$ & $\begin{array}{c}(6) \\
\text { Test3 } \\
\text { DUVOL }\end{array}$ \\
\hline$A T T_{t-1}$ & $\begin{array}{c}0.066^{* * *} \\
(3.02)\end{array}$ & $\begin{array}{c}0.069 * * * \\
(3.33)\end{array}$ & $\begin{array}{c}0.065^{* * *} \\
(3.00)\end{array}$ & $\begin{array}{c}0.054 * * \\
(2.48)\end{array}$ & $\begin{array}{c}0.069 * * * \\
(3.33)\end{array}$ & $\begin{array}{c}0.054 * * \\
(2.46)\end{array}$ \\
\hline OPTIMISM & & & $\begin{array}{c}0.034 * \\
(1.91)\end{array}$ & & & $\begin{array}{c}0.030^{*} \\
(1.70)\end{array}$ \\
\hline OPTIMISM $_{t-1}$ & & $\begin{array}{c}0.154 * * * \\
(8.77)\end{array}$ & & & $\begin{array}{c}0.154 * * * \\
(8.77)\end{array}$ & \\
\hline$S I G M A_{t-1}$ & & $\begin{array}{c}-0.162 * * \\
(-2.16)\end{array}$ & & & $\begin{array}{c}-0.162 * * \\
(-2.16)\end{array}$ & \\
\hline$R E T_{t-1}$ & $\begin{array}{c}-0.068 * * * \\
(-2.67)\end{array}$ & $\begin{array}{l}-0.035 \\
(-0.51)\end{array}$ & $\begin{array}{c}-0.071 * * * \\
(-2.80)\end{array}$ & $\begin{array}{c}-0.044 * \\
(-1.73)\end{array}$ & $\begin{array}{l}-0.035 \\
(-0.51)\end{array}$ & $\begin{array}{l}-0.047^{*} \\
(-1.85)\end{array}$ \\
\hline$S I Z E_{t-1}$ & & $\begin{array}{c}0.221 * * * \\
(2.63)\end{array}$ & & & $\begin{array}{c}0.221 * * * \\
(2.63)\end{array}$ & \\
\hline$L E V_{t-1}$ & $\begin{array}{l}-0.019 \\
(-0.42)\end{array}$ & $\begin{array}{c}-0.176^{* * * *} \\
(-3.48)\end{array}$ & $\begin{array}{l}-0.014 \\
(-0.31)\end{array}$ & $\begin{array}{l}-0.042 \\
(-0.90)\end{array}$ & $\begin{array}{c}-0.176 * * * \\
(-3.48)\end{array}$ & $\begin{array}{l}-0.037 \\
(-0.80)\end{array}$ \\
\hline$L_{I Q U I D I T Y}{ }_{t-1}$ & & $\begin{array}{l}0.049 \\
(1.45)\end{array}$ & & & $\begin{array}{l}0.049 \\
(1.45)\end{array}$ & \\
\hline$G R O W T H_{t-1}$ & & $\begin{array}{l}-0.020 \\
(-1.12)\end{array}$ & & & $\begin{array}{l}-0.020 \\
(-1.12)\end{array}$ & \\
\hline$A B A C C_{t-1}$ & $\begin{array}{l}-0.004 \\
(-0.22)\end{array}$ & $\begin{array}{c}0.045^{* * *} \\
(2.59)\end{array}$ & $\begin{array}{l}-0.005 \\
(-0.26)\end{array}$ & $\begin{array}{l}0.004 \\
(0.21)\end{array}$ & $\begin{array}{c}0.045^{* * * *} \\
(2.59)\end{array}$ & $\begin{array}{l}0.003 \\
(0.18)\end{array}$ \\
\hline$N C S K E W_{t-1}$ & $\begin{array}{c}-0.127 * * * \\
(-7.33)\end{array}$ & & $\begin{array}{c}-0.128 * * * \\
(-7.36)\end{array}$ & & & \\
\hline$T U R N O V E R_{t-1}$ & $\begin{array}{l}-0.024 \\
(-1.21)\end{array}$ & & $\begin{array}{l}-0.024 \\
(-1.22)\end{array}$ & $\begin{array}{l}-0.021 \\
(-1.07)\end{array}$ & & $\begin{array}{l}-0.022 \\
(-1.08)\end{array}$ \\
\hline$B M_{t-1}$ & $\begin{array}{c}-0.291 * * * \\
(-6.10)\end{array}$ & & $\begin{array}{c}-0.293 * * * \\
(-6.14)\end{array}$ & $\begin{array}{c}-0.307 * * * \\
(-6.40)\end{array}$ & & $\begin{array}{c}-0.309 * * * \\
(-6.43)\end{array}$ \\
\hline$R O A_{t-1}$ & $\begin{array}{l}-0.016 \\
(-0.53)\end{array}$ & & $\begin{array}{l}-0.020 \\
(-0.66)\end{array}$ & $\begin{array}{l}-0.029 \\
(-0.93)\end{array}$ & & $\begin{array}{l}-0.032 \\
(-1.05)\end{array}$ \\
\hline$D U V O L_{t-1}$ & & & & $\begin{array}{c}-0.149 * * * \\
(-8.71)\end{array}$ & & $\begin{array}{c}-0.150 * * * \\
(-8.74)\end{array}$ \\
\hline CONSTANT & $\begin{array}{l}-0.128 \\
(-0.99)\end{array}$ & $\begin{array}{l}0.135 \\
(1.08)\end{array}$ & $\begin{array}{l}-0.133 \\
(-1.03)\end{array}$ & $\begin{array}{l}-0.044 \\
(-0.34)\end{array}$ & $\begin{array}{l}0.135 \\
(1.08)\end{array}$ & $\begin{array}{l}-0.048 \\
(-0.37)\end{array}$ \\
\hline $\begin{array}{c}\text { YEAR } \\
\text { STATE } \\
\text { INDUSTRY }\end{array}$ & $\begin{array}{l}\text { Control } \\
\text { Control } \\
\text { Control }\end{array}$ & $\begin{array}{l}\text { Control } \\
\text { Control } \\
\text { Control }\end{array}$ & $\begin{array}{l}\text { Control } \\
\text { Control } \\
\text { Control }\end{array}$ & $\begin{array}{l}\text { Control } \\
\text { Control } \\
\text { Control }\end{array}$ & $\begin{array}{l}\text { Control } \\
\text { Control } \\
\text { Control }\end{array}$ & $\begin{array}{l}\text { Control } \\
\text { Control } \\
\text { Control }\end{array}$ \\
\hline $\begin{array}{c}\text { Observations } \\
\text { R-squared }\end{array}$ & $\begin{array}{l}4,062 \\
0.090\end{array}$ & $\begin{array}{l}4,062 \\
0.065\end{array}$ & $\begin{array}{l}4,062 \\
0.091\end{array}$ & $\begin{array}{l}4,062 \\
0.092\end{array}$ & $\begin{array}{l}4,062 \\
0.065\end{array}$ & $\begin{array}{l}4,062 \\
0.092\end{array}$ \\
\hline
\end{tabular}

$* * * \mathrm{p}<0.01, * * \mathrm{p}<0.05, * \mathrm{p}<0.1$ 
This article also reduced the sample size by selecting samples from 2016 to 2019 to verify the conclusions from the regression. The robustness results of the reduced sample size are presented in Table 6. According to these results, the conclusions of the tests were consistent with previous results suggesting that the research findings were robust.

Table 6. Robustness results of reduced sample size

\begin{tabular}{|c|c|c|c|c|c|c|}
\hline VARIABLES & $\begin{array}{c}(1) \\
\text { Test1 } \\
\text { NCSKEW }\end{array}$ & $\begin{array}{c}(2) \\
\text { Test2 } \\
\text { OPTIMISM }\end{array}$ & $\begin{array}{c}(3) \\
\text { Test3 } \\
N C S K E W\end{array}$ & $\begin{array}{c}(4) \\
\text { Test1 } \\
D U V O L\end{array}$ & $\begin{array}{c}(5) \\
\text { Test } 2 \\
\text { OPTIMISM } \\
\end{array}$ & $\begin{array}{c}(6) \\
\text { Test3 } \\
\text { DUVOL }\end{array}$ \\
\hline$A T T_{t-1}$ & $\begin{array}{c}0.080 * * \\
(2.03)\end{array}$ & $\begin{array}{c}0.072 * * \\
(2.02)\end{array}$ & $\begin{array}{c}0.076^{*} \\
(1.93)\end{array}$ & $\begin{array}{c}0.059 * * \\
(2.30)\end{array}$ & $\begin{array}{c}0.072 * * \\
(2.02)\end{array}$ & $\begin{array}{c}0.060 * * \\
(2.30)\end{array}$ \\
\hline OPTIMISM & & & $\begin{array}{c}0.102 * * * \\
(3.59)\end{array}$ & & & $\begin{array}{c}0.035^{*} \\
(1.65)\end{array}$ \\
\hline OPTIMISM $M_{t-1}$ & & $\begin{array}{c}-0.069 * * \\
(-2.43)\end{array}$ & & & $\begin{array}{c}-0.069 * * \\
(-2.43)\end{array}$ & \\
\hline$S I G M A_{t-1}$ & & $\begin{array}{l}-0.036 \\
(-0.27)\end{array}$ & & & $\begin{array}{l}-0.036 \\
(-0.27)\end{array}$ & \\
\hline$R E T_{t-1}$ & $\begin{array}{l}-0.017 \\
(-0.42)\end{array}$ & $\begin{array}{l}0.073 \\
(0.58)\end{array}$ & $\begin{array}{l}-0.028 \\
(-0.70)\end{array}$ & $\begin{array}{l}0.047 \\
(1.64)\end{array}$ & $\begin{array}{l}0.073 \\
(0.58)\end{array}$ & $\begin{array}{l}0.045 \\
(1.57)\end{array}$ \\
\hline$S I Z E_{t-1}$ & & $\begin{array}{l}0.155 \\
(0.85)\end{array}$ & & & $\begin{array}{l}0.155 \\
(0.85)\end{array}$ & \\
\hline$L E V_{t-1}$ & $\begin{array}{l}-0.124 \\
(-1.34)\end{array}$ & $\begin{array}{c}-0.390 * * * \\
(-3.88)\end{array}$ & $\begin{array}{l}-0.086 \\
(-0.93)\end{array}$ & $\begin{array}{l}0.009 \\
(0.30)\end{array}$ & $\begin{array}{c}-0.390 * * * \\
(-3.88)\end{array}$ & $\begin{array}{l}0.010 \\
(0.34)\end{array}$ \\
\hline$L_{I Q U I D I T Y}{ }_{t-I}$ & & $\begin{array}{l}0.068 \\
(1.07)\end{array}$ & & & $\begin{array}{l}0.068 \\
(1.07)\end{array}$ & \\
\hline$G R O W T H_{t-1}$ & & $\begin{array}{l}-0.033 \\
(-1.22)\end{array}$ & & & $\begin{array}{l}-0.033 \\
(-1.22)\end{array}$ & \\
\hline$A B A C C_{t-1}$ & $\begin{array}{l}0.015 \\
(0.57)\end{array}$ & $\begin{array}{l}0.030 \\
(1.12)\end{array}$ & $\begin{array}{l}0.012 \\
(0.45)\end{array}$ & $\begin{array}{l}-0.005 \\
(-0.25)\end{array}$ & $\begin{array}{l}0.030 \\
(1.12)\end{array}$ & $\begin{array}{l}-0.007 \\
(-0.30)\end{array}$ \\
\hline$N C S K E W_{t-1}$ & $\begin{array}{c}-0.280 * * * \\
(-10.92)\end{array}$ & & $\begin{array}{c}-0.283 * * * \\
(-11.07)\end{array}$ & & & \\
\hline$T_{U R N O V E R_{t-1}}$ & $\begin{array}{c}-0.053 * \\
(-1.72)\end{array}$ & & $\begin{array}{c}-0.056^{*} \\
(-1.81)\end{array}$ & $\begin{array}{l}0.026 \\
(0.99)\end{array}$ & & $\begin{array}{l}0.027 \\
(0.99)\end{array}$ \\
\hline$B M_{t-1}$ & $\begin{array}{c}-0.549 * * * \\
(-6.38)\end{array}$ & & $\begin{array}{c}-0.541 * * * \\
(-6.31)\end{array}$ & $\begin{array}{c}-0.124 * * * \\
(-3.61)\end{array}$ & & $\begin{array}{c}-0.121 * * * \\
(-3.50)\end{array}$ \\
\hline$R O A_{t-1}$ & $\begin{array}{l}-0.021 \\
(-0.44)\end{array}$ & & $\begin{array}{l}-0.037 \\
(-0.78)\end{array}$ & $\begin{array}{c}0.077 * * * \\
(2.79)\end{array}$ & & $\begin{array}{c}0.082 * * * \\
(2.83)\end{array}$ \\
\hline$D U V O L_{t-1}$ & & & & $\begin{array}{l}-0.003 \\
(-0.12)\end{array}$ & & $\begin{array}{l}-0.003 \\
(-0.16)\end{array}$ \\
\hline CONSTANT & $\begin{array}{c}-0.213 * * * \\
(-4.06)\end{array}$ & $\begin{array}{c}-0.168 * * * \\
(-4.67)\end{array}$ & $\begin{array}{c}-0.196 * * * \\
(-3.73)\end{array}$ & $\begin{array}{l}0.191 \\
(1.01)\end{array}$ & $\begin{array}{c}-0.168 * * * \\
(-4.67)\end{array}$ & $\begin{array}{l}0.185 \\
(0.92)\end{array}$ \\
\hline $\begin{array}{c}\text { YEAR } \\
\text { STATE } \\
\text { INDUSTRY }\end{array}$ & $\begin{array}{l}\text { Control } \\
\text { Control } \\
\text { Control }\end{array}$ & $\begin{array}{l}\text { Control } \\
\text { Control } \\
\text { Control }\end{array}$ & $\begin{array}{l}\text { Control } \\
\text { Control } \\
\text { Control }\end{array}$ & $\begin{array}{l}\text { Control } \\
\text { Control } \\
\text { Control }\end{array}$ & $\begin{array}{l}\text { Control } \\
\text { Control } \\
\text { Control }\end{array}$ & $\begin{array}{l}\text { Control } \\
\text { Control } \\
\text { Control }\end{array}$ \\
\hline $\begin{array}{c}\text { Observations } \\
\text { R-squared }\end{array}$ & $\begin{array}{l}2,031 \\
0.182\end{array}$ & $\begin{array}{l}2,031 \\
0.059\end{array}$ & $\begin{array}{l}2,031 \\
0.190\end{array}$ & $\begin{array}{l}2,031 \\
0.070\end{array}$ & $\begin{array}{l}2,031 \\
0.059\end{array}$ & $\begin{array}{l}2,031 \\
0.071\end{array}$ \\
\hline
\end{tabular}

$* * * \mathrm{p}<0.01, * * \mathrm{p}<0.05, * \mathrm{p}<0.1$ 


\subsection{Further analysis}

In comparison to ordinary investors, institutional investors are more professional in investments and have more advantages in collecting and interpreting information. Their investment choices and trading behaviors may result in investors' following behaviors. Therefore, the higher the institutional investor attention, the higher the overall attention. High attention tends to drive up stock prices, thus leading to a higher risk of price crash. High institutional investor attention would also enhance the impact of analyst optimism bias on the immediate response of the market. Analysts would be more inclined to issue optimism earnings forecasts. This shows that institutional investor attention tends to reinforce analyst optimism bias.

The shareholding ratio of institutional investors was used to measure the attention of institutional investors while IO was the independent variable. The correlations among institutional investor attention, analyst optimism bias, and stock price crash risk were further tested (in Table 7). Similar to ordinary investors, institutional investor attention would also aggravate crash risk and has a positive influence on analyst optimism bias which plays a mediating role in the positive correlation between the two variables.

Table 7. Institutional investor attention, analyst optimism, and crash risk

\begin{tabular}{|c|c|c|c|c|c|c|}
\hline VARIABLES & $\begin{array}{c}(1) \\
\text { Test1 } \\
N C S K E W\end{array}$ & $\begin{array}{c}(2) \\
\text { Test2 } \\
\text { OPTIMISM } \\
\end{array}$ & $\begin{array}{c}(3) \\
\text { Test3 } \\
N C S K E W\end{array}$ & $\begin{array}{c}(4) \\
\text { Test1 } \\
D U V O L\end{array}$ & $\begin{array}{c}(5) \\
\text { Test2 } \\
\text { OPTIMISM } \\
\end{array}$ & $\begin{array}{c}(6) \\
\text { Test3 } \\
D U V O L\end{array}$ \\
\hline$I O_{t-1}$ & $\begin{array}{c}0.100 * * * \\
(3.53)\end{array}$ & $\begin{array}{c}0.047^{*} \\
(1.73)\end{array}$ & $\begin{array}{c}0.100 * * * \\
(3.52)\end{array}$ & $\begin{array}{c}0.104 * * * \\
(3.64)\end{array}$ & $\begin{array}{c}0.047^{*} \\
(1.73)\end{array}$ & $\begin{array}{c}0.104 * * * \\
(3.63)\end{array}$ \\
\hline OPTIMISM & & & $\begin{array}{l}0.034 * \\
(1.94)\end{array}$ & & & $\begin{array}{c}0.031 * \\
(1.73)\end{array}$ \\
\hline$N C S K E W_{t-1}$ & $\begin{array}{c}-0.130 * * * \\
(-7.48)\end{array}$ & & $\begin{array}{c}-0.131 * * * * \\
(-7.52)\end{array}$ & & & \\
\hline$T U R N O V E R_{t-1}$ & $\begin{array}{l}-0.018 \\
(-0.90)\end{array}$ & & $\begin{array}{l}-0.018 \\
(-0.91)\end{array}$ & $\begin{array}{l}-0.016 \\
(-0.79)\end{array}$ & & $\begin{array}{l}-0.016 \\
(-0.79)\end{array}$ \\
\hline$R E T_{t-1}$ & $\begin{array}{c}-0.062^{* *} \\
(-2.43)\end{array}$ & $\begin{array}{l}0.026 \\
(0.37)\end{array}$ & $\begin{array}{c}-0.066 * * \\
(-2.57)\end{array}$ & $\begin{array}{l}-0.036 \\
(-1.42)\end{array}$ & $\begin{array}{l}0.026 \\
(0.37)\end{array}$ & $\begin{array}{l}-0.040 \\
(-1.55)\end{array}$ \\
\hline$B M_{t-1}$ & $\begin{array}{c}-0.300^{* * * *} \\
(-6.38)\end{array}$ & & $\begin{array}{c}-0.302^{* * * *} \\
(-6.41)\end{array}$ & $\begin{array}{c}-0.311^{* * * *} \\
(-6.56)\end{array}$ & & $\begin{array}{c}-0.312 * * * \\
(-6.59)\end{array}$ \\
\hline$L E V_{t-1}$ & $\begin{array}{l}0.002 \\
(0.04)\end{array}$ & & $\begin{array}{l}0.007 \\
(0.15)\end{array}$ & $\begin{array}{l}-0.020 \\
(-0.43)\end{array}$ & & $\begin{array}{l}-0.015 \\
(-0.33)\end{array}$ \\
\hline$R O A_{t-1}$ & $\begin{array}{l}-0.002 \\
(-0.06)\end{array}$ & & $\begin{array}{l}-0.006 \\
(-0.20)\end{array}$ & $\begin{array}{l}-0.018 \\
(-0.58)\end{array}$ & & $\begin{array}{l}-0.021 \\
(-0.70)\end{array}$ \\
\hline$A B A C C_{t-1}$ & $\begin{array}{l}-0.002 \\
(-0.08)\end{array}$ & $\begin{array}{c}0.043^{* *} \\
(2.44)\end{array}$ & $\begin{array}{l}-0.002 \\
(-0.13)\end{array}$ & $\begin{array}{l}0.006 \\
(0.34)\end{array}$ & $\begin{array}{c}0.043^{* *} \\
(2.44)\end{array}$ & $\begin{array}{l}0.005 \\
(0.30)\end{array}$ \\
\hline OPTIMISM ${ }_{t-1}$ & & $\begin{array}{c}0.148^{* * * *} \\
(8.43)\end{array}$ & & & $\begin{array}{c}0.148 * * * \\
(8.43)\end{array}$ & \\
\hline$S I G M A_{t-1}$ & & $\begin{array}{l}-0.081 \\
(-1.10)\end{array}$ & & & $\begin{array}{l}-0.081 \\
(-1.10)\end{array}$ & \\
\hline$G R O W T H_{t-1}$ & & $\begin{array}{l}-0.015 \\
(-0.82)\end{array}$ & & & $\begin{array}{l}-0.015 \\
(-0.82)\end{array}$ & \\
\hline$D U V O L_{t-1}$ & & & & $\begin{array}{c}-0.153 * * * \\
(-8.90)\end{array}$ & & $\begin{array}{c}-0.154 * * * \\
(-8.93)\end{array}$ \\
\hline CONSTANT & $\begin{array}{l}-0.113 \\
(-0.87)\end{array}$ & $\begin{array}{l}0.167 \\
(1.33)\end{array}$ & $\begin{array}{l}-0.118 \\
(-0.91)\end{array}$ & $\begin{array}{l}-0.026 \\
(-0.20)\end{array}$ & $\begin{array}{l}0.167 \\
(1.33)\end{array}$ & $\begin{array}{l}-0.030 \\
(-0.23)\end{array}$ \\
\hline
\end{tabular}




\begin{tabular}{c|c|c|c|c|c|c|c|c|c|}
\hline YEAR & Control & Control & Control & Control & Control & Control \\
STATE & Control & Control & Control & Control & Control & Control \\
\hline INDUSTRY & Control & Control & Control & Control & Control & Control \\
\hline Observations & 4,062 & 4,062 & 4,062 & 4,062 & 4,062 & 4,062 \\
R-squared & 0.091 & 0.053 & 0.092 & 0.094 & 0.053 & 0.094 \\
\hline
\end{tabular}

$* * * \mathrm{p}<0.01, * * \mathrm{p}<0.05, * \mathrm{p}<0.1$

\section{Conclusion}

By using samples from China's A-shares listed companies from 2013 to 2019, this article constructed a comprehensive system to measure investor attention based on macro and micro perspectives, and conducted an empirical research on the correlations among investor attention, analyst optimism bias, and stock price crash risk. The results showed that investor attention would aggravate stock price crash risk and has a positive impact on analyst optimism bias. Meanwhile, analyst optimism bias plays an intermediary role in the positive correlation between investor attention and crash risk. These results are robust to a series of tests including substituting independent variables and reducing the sample size. Similar to ordinary investor attention, institutional investor attention also has both, direct and indirect positive effects on the crash risk.

\section{Disclosure statement}

The author declares no conflict of interest.

\section{References}

[1] Jin L, Myers S, 2006, R2 around the world: new theory and new tests. J Financ Econ, 79: 257-292.

[2] Francis J, Philbrick D, 1993, Analyst decisions as products of a multi-task environment. J Account Res, 31: 216-230.

[3] Huddart S, Lang M, Yetman MH, 2009, Volume and price patterns around a stock's 52-week highs and lows: theory and evidence. Manage Sci, 55: 16-31.

[4] Barber BM, Odean T, 2008, All that glitters: the effect of attention and news on the buying behavior of individual and institutional investors. Rev Financ Stud, 21: 785-818.

[5] Abreu D, Brunnermeier MK, 2003, Bubbles and crashes. Econometrica, 71: 173-204.

[6] Mola S, Guidolin M, 2009, Affiliated mutual funds and analyst optimism. J Financ Econ, 93: 108-137.

[7] Boni L, Womack, 2006, Analysts, industries, and price momentum. Financ Quant Anal, 41: 85-109.

[8] Loh RK, 2010, Investor inattention and the underreaction to stock recommendations. Financ Manage, 39: $1223-1252$.

[9] Kim JB, Li Y, Zhang L, 2011, Corporate tax avoidance and stock price crash risk: firm-level analysis. J Financ Econ, 100: 639-662.

[10] Fu J, Wu X, Liu Y, et al., 2021, Firm-specific investor sentiment and stock price crash risk. Financ Res Lett, 38: 101442.

[11] Wen F, Xu L, Chen B, et al., 2020, Heterogeneous institutional investors, short selling and stock price crash risk: evidence from china. Emerg Mark Financ Tr, 56: 2812-2825.

[12] Fama EF, French KR, 2015, A five-factor asset pricing model. J Financ Econ, 116: 1-22.

[13] Jackson AR, 2005, Trade generation, reputation, and sell-side analysts. J Financ, 60: 673-717. 